\title{
Anomaly cancellation for anisotropic lattice fields with extra dimensions
}

\author{
Stam Nicolis* \\ CNRS-Laboratoire de Mathématiques et Physique Théorique (UMR 6083) \\ Fédération de Recherche "Denis Poisson" (FR 2964) \\ Université "François Rabelais” de Tours \\ Parc Grandmont, 37200 Tours, France \\ E-mail: Stam.Nicolis@lmpt.univ-tours.fr
}

\begin{abstract}
The current flow from the bulk is due to the anomaly on the brane-but the absence of current flow is not, necessarily due to anomaly cancellation, but to the absence of the chiral zero modes themselves, due to the existence of the layered phase. This can be understood in terms of the difference between the Chern-Simons terms in three and five dimensions. Thus the anomaly cancellation in four dimensions, which is essential for shielding the boundary from quantum effects within the bulk, makes sense only along the transition line between the layered and the Coulomb phase, which, in turn, requires the presence of a compact $U(1)$ factor for the gauge group.
\end{abstract}

The XXVII International Symposium on Lattice Field Theory - LAT2009

July 26-31 2009

Peking University, Beijing, China

${ }^{*}$ Speaker. 
Domain wall [1] and overlap [2] fermions allow the realization of chiral symmetry on the lattice [3]. The key ingredients, that lead to evading the no-go theorem [4], are the defects that extend in extra dimensions. The chiral zero modes are localized along appropriate boundaries. For a consistent quantum theory we would like that the boundary theory be protected from quantum effects within the bulk.

Since the fermions are coupled to gauge fields, there will generically be anomalies [5]. These appear as current flow to and from the bulk. They also appear as "edge currents". Therefore anomalies should be properly cancelled [6]. Anomaly cancellation has been studied for the chiral Schwinger model [7], i.e. a three-dimensional bulk with two-dimensional boundaries, but not for the five-dimensional case. The object of this note is to point out some essential differences between the two cases. These differences are also relevant for attempts at detecting extra dimensions [8].

In three dimensions the relevant contribution to the anomaly is the vacuum polarization diagram (cf. Fig. 1) which is well-known to provide a "topological" mass term for the gauge

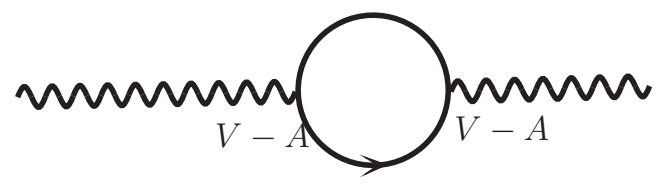

Figure 1: The contribution to the anomaly in $D=2$.

field $[9,10]$. In five dimensions the relevant contribution is the well-known triangle diagram (cf. Fig. 2) which does not provide a mass for the gauge field-it is an interaction term. In fact this is

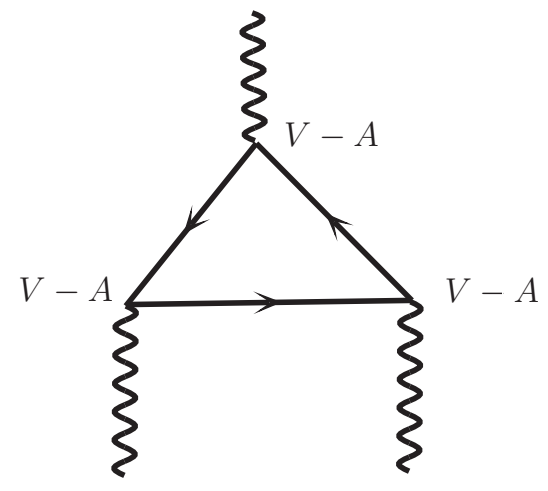

Figure 2: The contribution to the anomaly in $D=4$.

the crucial difference: in three dimensions the anomaly is a "soft" term, whereas in five dimensions it is a "hard" term. This was in fact noticed in refs. [11, 12] for the four-dimensional anomaly in that the renormalization constant for the axial current could not be expressed in terms of the renormalization constant of mass term and that of the wave function renormalization constant of the fermion, but was an independent quantity.

In fact it is possible to understand this in another way. The anisotropy of the defects implies [1] that the gauge couplings will generically be different along the boundary $(\beta)$ and towards the bulk 
$\left(\beta^{\prime}\right)$. The action on the lattice will then take the form (for the five-dimensional case)

$$
S=\beta \sum_{n} \sum_{\mu<v}\left(1-\operatorname{Re} U_{\mu v}(n)\right)+\beta^{\prime} \sum_{n} \sum_{\mu}\left(1-\operatorname{Re} U_{\mu 5}(n)\right)+S_{\text {fermions }}
$$

In the three-dimensional case the isotropic theory, $\beta=\beta^{\prime}$ is renormalizable (in fact it is superrenormalizable). This means that, once anomalies are cancelled, the two-dimensional theory is protected from quantum bulk effects. In the five-dimensional case, however, the isotropic theory is not renormalizable-it cannot be made insensitive to cutoff effects, which renders the decoupling of its boundaries questionable. However, the anisotropic theory may have a layered phase [13]: and along the transition line between this phase and the bulk Coulomb phase it seems that a continuum theory could be defined, since this transition seems to be second order [14, 15] (cf. Fig. 3) Therefore

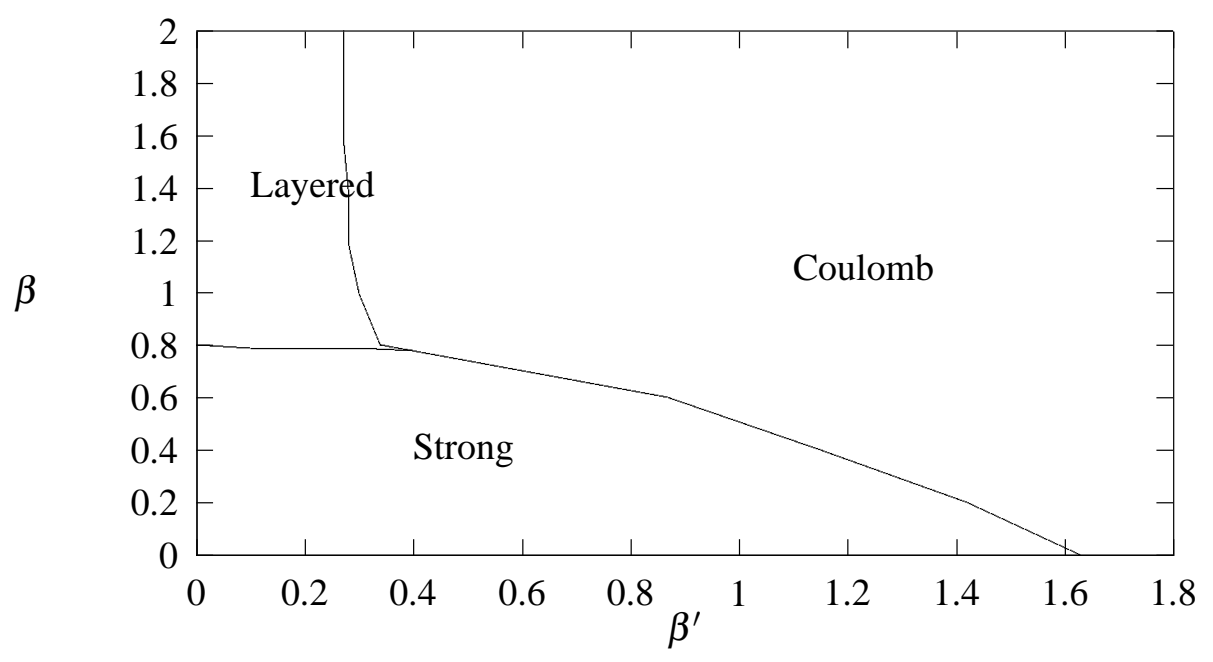

Figure 3: The phase diagram of the five-dimensional $U(1)$ theory in the $\beta-\beta^{\prime}$ plane.

it is for gauge couplings along this line that the anomaly cancellation can be realized. Indeed, the current, that carries the anomaly flow from and to the bulk plays a double rôle: on the one hand it is a component of the bulk vector current; on the other hand it is the axial current density from the point of view of the boundary. The chiral charge on the boundary has absolute value that of the (vector) bulk charge and sign the chirality at the boundary. Thus these models have a gauged chiral symmetry.

This current can indeed be computed [14] and displays the following behavior (cf. Fig. 4) It is noteworthy that this current vanishes within the layered phase and is non-zero in the bulk Coulomb phase. The reason it does not vanish in the Coulomb phase is a manifestation of the anomaly-the reason it vanishes in the layered phase is not anomaly cancellation, but, rather, that there isn't any chiral zero mode that can absorb or emit the charge [16].

The model we studied was based on the compaact formulation of the $U(1)$ gauge group. The reason is that this group is the only one that presents both a Coulomb phase and a confining phase, that are separated by a phase transition. This is essential to the possibility of defining the layered 


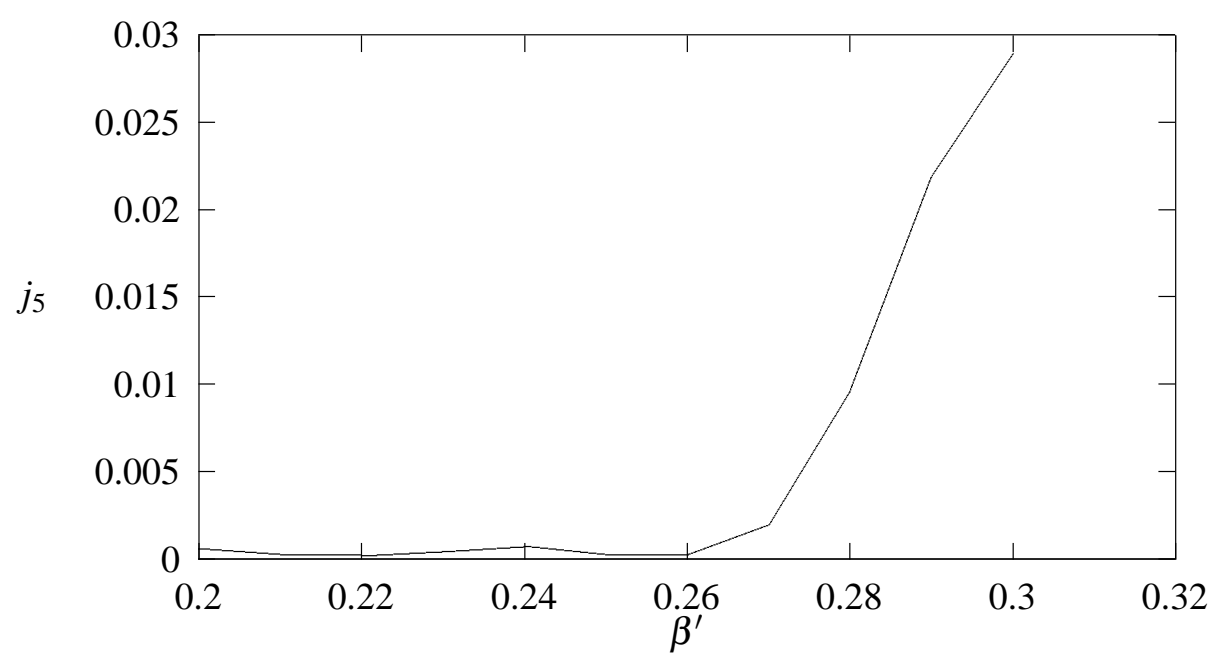

Figure 4: The current along the extra dimension, for $\beta=1.2$, as a function of $\beta^{\prime}$.

phase intrinsically [17]. Anisotropic Yang-Mills theories are harder to study (cf. ref. [18] for a study of the decoupling effects in a model with $S U(2)$ gauge group; it will be interesting to compare this with the case of $U(2)=S U(2) \otimes U(1)$, which should provide a much cleaner signal, since the $U(1)$ factor will trigger the layered to Coulomb phase transition along which the anomaly cancellation should be realized. Studies with $S U(3)$ have been attempted [19] but did not take into account this difference, so it is hard to see exactly how the layer is defined.).

In conclusion, the difference between the anomaly terms in three and five dimensions is crucial in understanding the difference in the physics between the two cases. In three dimensions the anomaly term is a mass term and both the bulk and the boundary theories are renormalizable at the isotropic point, where anomaly cancellation can be imposed. In five dimensions the anomaly term is an interaction vertex (whose, dimensionless, coefficient may be related to the ratio of the, dimensionful, coupling constants in the boundary and towards the bulk). Anomaly cancellation cannot take place along the isotropic line,since the bulk theory is not renormalizable there-the layered phase, however, leads to the possibility of shielding the boundary from the bulk along a line of strongly coupled fixed points, that may be also relevant in other contexts (e.g. [20]).

\section{Acknowledgments}

It is a pleasure to acknowledge discussions with S. M. Catterall, Ph. de Forcrand, L. Giusti and M. Panero.

\section{References}

[1] D. B. Kaplan, A Method for simulating chiral fermions on the lattice, Phys. Lett. B288 (1992) 342 [arXiv:hep-lat/9206013]. 
[2] R. Narayanan and H. Neuberger, Infinitely many regulator fields for chiral fermions, Phys. Lett. B302 (1993) 62 [arXiv: hep-lat/9212019].

[3] M. Lüscher, Lattice regularization of chiral gauge theories to all orders of perturbation theory, JHEP 0006 (2000) 028 [arXiv: hep-lat/0006014] .

[4] H. B. Nielsen and M. Ninomiya, Absence Of Neutrinos On A Lattice. 1. Proof By Homotopy Theory, Nucl. Phys. B185 (1981) 20 [Erratum-ibid. B195 (1982) 541]. H. B. Nielsen and M. Ninomiya, Absence Of Neutrinos On A Lattice. 2. Intuitive Topological Proof, Nucl. Phys. B193 (1981) 173.

[5] C. G. Callan and J. A. Harvey, Anomalies And Fermion Zero Modes On Strings And Domain Walls, Nucl. Phys. B250 (1985) 427.

[6] C. Bouchiat, J. Iliopoulos and Ph. Meyer, 'An Anomaly Free Version Of Weinberg's Model, Phys. Lett. 38B 519 (1972).

[7] K. Jansen; Chiral fermions and anomalies on a finite lattice, Phys. Lett. B288 (1992) 348 [arXiv: hep-lat/9206014] . M. Golterman, K. Jansen; D. B. Kaplan; Chern-Simons currents and chiral fermions on the lattice, Phys. Lett. B301 (1993) 219 [arXiv: hep-lat/9209003] . K. Jansen and M. Schmaltz, Critical momenta of lattice chiral fermions, Phys. Lett. B296 (1992) 374 [arXiv: hep-lat/9209002]. E. Poppitz and Y. Shang, Lattice chirality, anomaly matching, and more on the (non)decoupling of mirror fermions, [arXiv:0901.3402 [hep-lat] ].

[8] A. Boyarsky, O. Ruchayskiy and M. Shaposhnikov, Anomalies as a signature of extra dimensions, Phys. Lett. B626 (2005) 184 [arXiv: hep-ph/0507195].

[9] S. Deser, R. Jackiw, S. Templeton, Topologically massive gauge theories, Annals Phys. 140 (1982) 372 [Erratum-ibid. 185 (1988 APNYA,281,409-449.2000) 406.1988 APNYA,281,409].

[10] S. R. Coleman and B. Hill, No More Corrections To The Topological Mass Term In QED In Three-Dimensions, Phys. Lett. B159 (1985) 184.

[11] S. L. Adler, Axial vector vertex in spinor electrodynamics, Phys. Rev. 177 (1969) 2426.

[12] G. Preparata and W. I. Weisberger, Ultraviolet divergences in radiative corrections to weak decays, Phys. Rev. 175 (1968) 1965.

[13] Y. K. Fu and H. B. Nielsen, A Layer Phase In A Nonisotropic U(1) Lattice Gauge Theory: Dimensional Reduction A New Way, Nucl. Phys. B236 (1984) 167. Some Properties Of The Layer Phase, Nucl. Phys. B254 (1985) 127.

[14] A. Hulsebos, C. P. Korthals-Altes and S. Nicolis, Gauge Theories with a Layered Phase, Nucl. Phys. B450 (1995) 437 [hep-th/9406003] ; A. Hulsebos, Anisotropic gauge theories: A Numerical study of the Fu-Nielsen model, Nucl. Phys. Proc. Suppl. 42 (1995) 618, [hep-lat/9412031];

[15] K. Farakos and S. Vrentzos, Establishment of the Coulomb law in the layer phase of a pure U(1) lattice gauge theory, Phys. Rev. D77 (2008) 094511 [arXiv:0801.3722 [hep-lat]].

[16] C. P. Korthals-Altes, S. Nicolis and J. Prades, Chiral defect fermions and the layered phase, Phys. Lett. B316 (1993) 339 [arXiv: hep-lat/9306017].

[17] S. Nicolis, Layered Phase Investigations , PoS LAT2007 (2007) 273 [ arXiv: 0710.1714 [hep-lat] ].

[18] A. Kurkela, parallel talk, these proceedings. 
[19] Y. K. Fu, L. X. Huang and D. X. Zhang, The Layer phase in the nonisotropic Abelian and nonAbelian gauge model, Phys. Lett. $B 335$ (1994) 65. G. L. Wang and Y. K. Fu, The layer phase in the non-isotropic SU(3) gauge model at finite temperature, [arXiv: hep-th/0101146] . K. Petrov, Layer World: Living on a layer in 5D SU(3), [arXiv:0911.1071 [hep-lat] ]; these proceedings.

[20] A. Deuzeman, M. P. Lombardo and E. Pallante, Evidence for a conformal phase in SU(N) gauge theories, [arXiv:0904.4662 [hep-ph] ]. E. Pallante, plenary talk; these proceedings. D. Dorigoni and S. Rychkov, Scale Invariance + Unitarity $=>$ Conformal Invariance?, [arXiv:0910.1087 [hep-th]]. 